\title{
Red Blood Cell Distribution Width as a 5-Year Prognostic Marker in Patients Submitted to Carotid Endarterectomy
}

\author{
Luís Duarte-Gamas ${ }^{a, b}$ António Pereira-Neves ${ }^{a, b}$, c Filipa Jácome ${ }^{a, b}$ \\ Mariana Fragão-Marques ${ }^{b, d}$, e Ricardo P. Vaz ${ }^{b, f} \quad$ Jose Paulo Andrade ${ }^{b, f}$ \\ João P. Rocha-Neves ${ }^{a}$, b, c \\ a Department of Angiology and Vascular Surgery, Centro Hospitalar Universitário de \\ São João, Porto, Portugal; ${ }^{b}$ Department of Surgery and Physiology, Faculdade de Medicina \\ da Universidade do Porto, Porto, Portugal; ' $D$ epartment of Biomedicine, Unit of Anatomy, \\ Faculdade de Medicina da Universidade do Porto, Porto, Portugal; ${ }^{\mathrm{d}}$ Department of Clinical \\ Pathology, Centro Hospitalar Universitário de São João, Porto, Portugal; ${ }^{e}$ Cardiovascular \\ R\&D Unit, Faculdade de Medicina da Universidade do Porto, Porto, Portugal; ${ }^{f}$ Center for \\ Health Technology and Services Research (CINTESIS), Porto, Portugal
}

\section{Keywords}

Endarterectomy · Carotid · Prognosis - Erythrocyte Indices - Major cardiovascular events . Mortality

\section{Abstract}

Objective: Patients submitted to carotid artery endarterectomy (CEA) have a long-term risk of major adverse cardiovascular events (MACE) of 6-9\% at 2 years. Hematological parameters have been shown to have a predictive function in atherosclerotic diseases, namely the red blood cell distribution width-coefficient of variation (RDW-CV). This parameter has been associated with worse outcomes such as myocardial infarction (MI), stroke, and all-cause mortality. This study aims to evaluate the potential role of preoperative hematologic parameters such as RDW-CV in predicting perioperative and long-term cardiovascular adverse events and mortality in patients submitted to CEA. Methods: From January 2012 to January 2019, 180 patients who underwent CEA with regional anesthesia in a tertiary care and referral center were selected from a prospective cohort database. Blood samples were collected preoperatively 2 weeks before admission, including a full blood count. The primary outcome included long-term MACE. Secondary outcomes included all-cause mortality, stroke, MI, acute heart failure, and major adverse limb events (MALE). Results: At baseline, 27.2\% of patients had increased RDW-CV. Increased RDW-CV was independently associated with baseline hemoglobin (adjusted odds ratio [aOR] $0.715,95 \% \mathrm{Cl} 0.588-0.869, p=0.001)$ and atrial fibrillation $(\mathrm{aOR}$ 
Duarte-Gamas et al.: RDW as a Long-Term Prognostic Marker after Carotid Endarterectomy

4.028, 95\% Cl 1.037-15.639, $p=0.001$ ). After a median follow-up of 50 months, log-rank univariate analysis of RDW-CV demonstrated a significant association between increased RDWCV and long-term all-cause mortality (log-rank <0.001), MACE (log-rank <0.001), and MI (logrank $=0.017)$. After multivariate Cox regression analysis, increased RDW-CV was associated with increased long-term mortality (adjusted hazard ratio [aHR] 2.455, 95\% Cl 1.231-4.894, $p=0.011)$ and MACE (aHR 2.047, 95\% Cl 1.202-3.487, $p=0.008$ ). A decreased hemoglobin to platelet ratio (aHR 2.650e-8, 95\% Cl 9.049e-15 to 0.078, $p=0.019$ ) was also associated with all-cause mortality. Conclusion: RDW is a widely available and low-cost marker that independently predicts long-term mortality, MACE, and MI after CEA. This biomarker could prove useful in assessing which patients would likely benefit from CEA in the long term.

(C) 2020 The Author(s)

Published by S. Karger AG, Basel

\section{Introduction}

Carotid artery revascularization has proven benefits and is indicated in patients with symptomatic carotid stenosis (CS) $>50 \%$, based on the NASCET method for measuring the degree of stenosis, and selected patients with asymptomatic CS $>60 \%$ (NASCET method [1]) or $80 \%$ (ECST method [2]) with acceptable perioperative risk and a 5-year life expectancy. While the benefit of carotid artery endarterectomy (CEA) is consensual in symptomatic CS, it is less well established in the asymptomatic CS [3]. Large randomized controlled clinical trials, such as the Asymptomatic Carotid Atherosclerosis Study (ACST) and the Asymptomatic Carotid Surgery Trial 1 (ACST-1), compared CEA with medical treatment in patients with asymptomatic CS $>60 \%[4,5]$. Both trials found a reduction in stroke or death at 5 years of follow-up in the CEA arm compared to patients on medical treatment alone. Meanwhile, evidence and clinical practice concerning medical treatment have evolved since the end of these trials. This benefit of CEA in asymptomatic patients is mostly hampered by long-term major cardiovascular adverse events (MACE). The 2-year MACE rates after CEA have been described as 6-9\% [6, 7]. An exhaustive preoperative risk assessment is, therefore, paramount in order to select which patients with asymptomatic CS may benefit from surgery.

The prognostic ability of hematological parameters in atherosclerotic diseases has been rarely described, particularly the red blood cell distribution width (RDW), a parameter which reflects erythrocyte size distribution and is readily available in a full blood count. Most automated hematological cell counters calculate it as a coefficient of variation (CV), and the final result is expressed as a percentage, generally comprised between 12 and 15\% [8].

Increased RDW-CV was initially acknowledged as a marker of iron deficiency [9]. However, several additional potential mechanisms have been proposed for the increase of RDW-CV, such as nutritional deficiency (vitamin B12, folic acid), bone marrow depression, or inflammation, which leads to the extension of the red blood cell lifespan [10]. Recent studies have pointed out a link among RDW, diabetes mellitus, and inflammation. RDW-CV is also strongly associated with the incidence of stroke and myocardial infarction (MI) [11]. Furthermore, it seems to be a reliable and independent predictor of mortality in coronary artery disease (CAD) and ischemic stroke $[12,13]$.

Considering this prognostic ability of RDW-CV, it can be hypothesized that it could prove useful in predicting postoperative and long-term outcomes after CEA and so aid in the decisionmaking process of patient selection. This study aims to evaluate the potential role of preoperative hematologic parameters such as RDW-CV in predicting long-term MACE in patients submitted to CEA. As a secondary outcome, this study aimed to determine the prognostic value of RDW-CV concerning crucial long-term adverse outcomes, including MI, stroke, acute heart failure (AHF), and all-cause mortality in patients undergoing CEA with regional anesthesia.

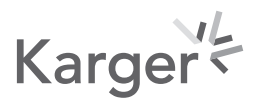




\section{Methods}

\section{Study Sample}

From January 2012 to January 2019, 180 patients who underwent CEA with regional anesthesia for CS in a tertiary care and referral center were consecutively selected from a prospectively maintained cohort database, and a post hoc analysis was performed. Patients were evaluated preoperatively by a vascular surgeon, and an anesthesiologist and their demographic and clinical data were collected. Patients were included as long as a preoperative full blood count was available in the 2 weeks preceding the CEA. Patients were excluded if CEA was performed under general anesthesia or if a synchronous myocardial revascularization surgery was performed.

All patients were under statin and single antiplatelet therapy for at least 2 days before surgery. Symptomatic CS was defined as having symptoms of stroke/transient ischemic attack in the previous 6 months before CS was detected and quantified by a duplex ultrasound exam or a computed tomography angiogram (NASCET) [14] or an additional duplex ultrasound exam performed by a different independent operator. This study was reported according to the Strengthening the Reporting of Cohort Studies in Surgery (STROCSS) 2019 criteria [15]. Patient informed consent was handled according to the observational nature of the data, and all data processing was anonymous.

\section{Analytical Parameters}

Blood samples were collected preoperatively at the time of admission. All patients had at least one blood sample taken and analyzed within 2 weeks before surgery. Analytical parameters included a complete blood count, serum creatinine and urea, electrolytes, and coagulation testing. Complete blood counts were performed with the Sysmex XE-2100D automated hematology analyzer (Sysmex Corporation, Kobe, Japan), with reference values for RDW-CV between 11 and 16\%.

\section{Outcome Assessment}

Patients were evaluated for the first 30 days after the surgical intervention, after 6 months, 1 year, and yearly after that. Follow-up included duplex ultrasound until 2 years of follow-up. The primary outcome was long-term MACE, defined as a composite outcome of non-fatal MI, AHF (onset of or de novo AHF), and all-cause mortality [6]. Secondary outcomes included all-cause mortality, stroke, MI, AHF, and major adverse limb events (MALE; defined as a composite outcome of major ischemic amputation, acute limb ischemia, and need for peripheral revascularization) [16].

\section{Statistical Analysis}

The necessary sample for a survival test was calculated using WinPepi ${ }^{\circledR} \mathrm{v} \cdot 11.65$, aiming for statistical power $(\beta)$ of $80 \%$ and a statistical significance of $\alpha<0.05$. The described MACE rate in long-term post-CEA patients is approximately $6 \%$ [6]. The described event rates after 5 years of follow-up in stroke patients with normal and increased RDW-CV varies between 49 and $69 \%$ and 69 and $88 \%$, respectively $[17,18]$. Based on these findings, an event rate difference of $20 \%$ between groups was established as reasonable. The estimated sample size was 147.

Statistical testing was performed with SPSS Statistics for Windows v.25.0 (IBM Corp., Armonk, NY, USA). The C-statistic was analyzed to determine the optimal cutoff of RDW-CV for the prediction of MACE.

Patients with increased RDW-CV ( $>13.95 \%$, according to Youden's J statistic) were compared with patients with low RDW-CV $(<13.95 \%)$ with respect to baseline demographics

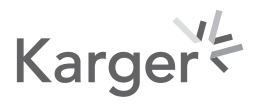


Table 1. Patient demographics and comorbidities

\begin{tabular}{|c|c|c|c|c|}
\hline & $\begin{array}{l}\text { RDW-CV }<13.95 \% \\
(n=131)\end{array}$ & $\begin{array}{l}\text { RDW-CV } \geq 13.95 \% \\
(n=49)\end{array}$ & $p$ value & $\begin{array}{l}\text { Multivariate } \\
\text { aOR 95\% CI }\end{array}$ \\
\hline Age, years & $69.63 \pm 9.03$ & $71.31 \pm 8.52$ & 0.263 & \\
\hline Sex, male & $103(78.6)$ & $41(83.7)$ & 0.451 & \\
\hline Hypertension & $111(84.7)$ & $46(93.9)$ & 0.102 & $\mathrm{NC}$ \\
\hline Smoking history & $65(49.6)$ & $26(53.1)$ & 0.681 & \\
\hline Diabetes mellitus & $55(42.0)$ & $23(46.9)$ & 0.551 & \\
\hline Dyslipidemia & $113(86.3)$ & $42(85.7)$ & 0.925 & \\
\hline CKD & $13(9.9)$ & $12(24.5)$ & 0.012 & $\mathrm{NC}$ \\
\hline $\mathrm{BMI}>30$ & $27(20.6)$ & $5(10.2)$ & 0.104 & \\
\hline PAD & $25(19.1)$ & $17(34.7)$ & 0.028 & $\mathrm{NC}$ \\
\hline CAD & $40(30.5)$ & $21(42.9)$ & 0.120 & \\
\hline COPD & $18(13.7)$ & $5(10.2)$ & 0.527 & \\
\hline $\mathrm{CHF}$ & $12(9.2)$ & $10(20.4)$ & 0.04 & $\mathrm{NC}$ \\
\hline Atrial fibrillation & $5(3.8)$ & 7 (14.3) & 0.012 & $4.028(1.037-15.639)$ \\
\hline ASA II & $22(18.5)$ & $4(8.3)$ & 0.900 & \\
\hline ASA III & $85(71.4)$ & $44(91.7)$ & & \\
\hline ASA IV & $12(10.1)$ & 0 & & \\
\hline Asymptomatic & $68(51.9)$ & $32(65.3)$ & & \\
\hline Symptomatic & & & 0.093 & NC \\
\hline TIA & $11(8.4)$ & $6(12.2)$ & & \\
\hline Stroke & $52(39.7)$ & $11(22.4)$ & & \\
\hline $\mathrm{BB}$ & $39(31.2)$ & $16(33.3)$ & 0.801 & \\
\hline ACEI & $87(69.6)$ & 37 (77.1) & 0.328 & \\
\hline $\mathrm{CCB}$ & $47(37.6)$ & $19(39.6)$ & 0.810 & \\
\hline Thiazide diuretics & $56(44.8)$ & $21(43.8)$ & 0.901 & \\
\hline Hemoglobin, g/dL & $13.39 \pm 1.71$ & $12.39 \pm 2.07$ & 0.001 & $0.715(0.588-0.869)$ \\
\hline NLR & $2.305 \pm 1.13$ & $3.13 \pm 1.78$ & 0.004 & $\mathrm{NC}$ \\
\hline PLR & $118.24 \pm 56.73$ & $142.62 \pm 99.64$ & 0.111 & $\mathrm{NC}$ \\
\hline HPR & $0.066 \pm 0.022$ & $0.0625 \pm 0.025$ & 0.394 & \\
\hline
\end{tabular}

Data are presented as the mean \pm SD or $n(\%)$. Statistically significant results are highlighted in bold. aOR, adjusted odds ratio; 95\% CI, 95\% confidence interval; ASA, American Society of Anesthesiologists Physical Status Classification System; ACEI, angiotensin conversion enzyme inhibitor; BB, beta-blockers; CAD, coronary artery disease; CCB, calcium channel blockers; CHF, congestive heart failure; CKD, chronic kidney disease (creatinine $=1.5$ $\mathrm{mg} / \mathrm{dL}$ ); COPD, chronic obstructive pulmonary disease; HPR, hemoglobin to platelet ratio; PAD, peripheral artery disease; BMI, body mass index (obesity $>30$ ); NC, not confirmed on multivariate analysis; NLR, neutrophil to lymphocyte ratio; PLR, platelet to lymphocyte ratio; RDW-CV, red blood cell distribution width-coefficient of variation; TIA, transient ischemic attack.

and clinical characteristics. Univariate analysis was performed with $\chi^{2}$ and Fischer's exact tests for categorical data and the Student $t$ and Mann-Whitney U tests for continuous data. A $p$ value $<0.05$ was considered statistically significant for the analysis.

Multivariate logistic regression analysis was performed to determine independent clinical and demographic factors associated with increased RDW-CV. The stepwise dimension reduction method was used, and variables with $p<0.15$ were included, considering atherosclerotic morbidity for the last steps. The log-rank test was used to determine the effect of RDW-CV on long-term primary and secondary outcomes. Multivariate Cox regression analysis was performed for independent predictors of long-term MACE and all-cause mortality. 


\section{Results}

\section{Demographic Data}

The sample consisted of 180 patients ( $80 \%$ male) with a mean age of $70.1 \pm 8.9$ years. Forty-nine (27.2\%) patients had increased preoperative RDW-CV. There were no significant differences in age and gender between the RDW-CV groups. The median sample follow-up was 50 months (95\% CI 42.9-57.1).

Regarding comorbidities, increased RDW-CV was significantly associated with chronic kidney disease (CKD; creatinine >1.5 mg/dL; 9.9 vs. 24.5\%, $p=0.012$ ), peripheral artery disease (PAD; 19.1 vs. 34.7\%, $p=0.028$ ), congestive heart failure (CHF; 9.2 vs. 20.4\%, $p=$ 0.04 ), and atrial fibrillation ( 3.8 vs. $14.3 \%, p=0.012$ ). There were no significant associations between increased RDW-CV and CAD, body mass index $>30$, arterial hypertension (HTA), or carotid-related symptomatic status (Table 1 ).

After multivariate regression analysis, increased RDW-CV had a significant independent association with preoperative hemoglobin (adjusted odds ratio [aOR] 0.715, 95\% CI 0.5880.869 ) and atrial fibrillation (aOR 4.028, 95\% CI 1.037-15.639). Multivariate analysis did not confirm the association between increased RDW-CV and HTA, CHF, CKD, or PAD (Table 1).

\section{Predictive Ability of RDW-CV on Long-Term Outcomes}

Considering survival times, after univariate analysis, CKD (median survival time [MST] 50 vs. 49 months, $p=0.001$ ) and PAD (MST 57 vs. 49 months, $p=0.004$ ) were associated with a lower MST (Table 2). Log-rank univariate analysis of RDW-CV demonstrated a significant association between increased RDW-CV and long-term all-cause mortality (log-rank $<0.001$ ), MACE (log-rank <0.001), and MI (log-rank $=0.017$ ). No association was found between increased RDW-CV and stroke (log-rank $=0.208)$, AHF (log-rank $=0.934)$, or MALE (log-rank $=0.185$; Fig. 1). The C-statistic analysis and Youden's J index determined the optimal RDW-CV cutoff for determination of the primary outcome as 13.95\%.

RDW and other factors significantly $(p<0.15)$ associated with decreased MST or baseline demographics were included in the multivariate Cox regression model in order to adjust for confounding and assess the independent effect of these factors on the primary and secondary outcomes. After multivariate Cox regression analysis, long-term mortality was significantly higher in patients with CKD (adjusted hazard ratio [aHR] 2.904; 95\% CI 1.410-5.982, $p=$ 0.004), PAD (aHR 2.138; 95\% CI 1.066-4.292, $p=0.032$ ), decreased hemoglobin to platelet ratio (HPR; aHR 2.650e-8; 95\% CI 9.049e-15 to 0.078, $p=0.019$ ), and increased RDW-CV (aHR 2.455, 95\% CI 1.231-4.894, $p=0.011$; Table 3). Increased RDW-CV (aHR 2.047, 95\% CI $1.202-3.487, p=0.008$ ) and CKD (aHR 2.056, 95\% CI 1.117-3.786, $p=0.021$ ) were independently associated with long-term MACE (Table 3).

\section{Discussion}

The main findings of this study were the significant associations of increased RDW-CV with long-term MACE, MI, and long-term all-cause mortality in patients submitted to CEA. The association between RDW-CV and adverse events has been studied in many cardiovascular diseases. Increased RDW-CV is independently associated with long-term all-cause mortality in patients undergoing percutaneous coronary intervention [12], regardless of hemoglobin values.

Few studies have focused on the association between RDW-CV and carotid atherosclerosis. Cross-sectional studies have found an association between increased RDW-CV and carotid intima-media thickness [19]. In the Tromso study, increased RDW-CV was an inde- 

Diseases Extra

\begin{tabular}{l|l}
\hline DOI: 10.1159/000512587 & $\begin{array}{l}\text { (c) 2020 The Author(s). Published by S. Karger AG, Basel } \\
\text { www.karger.com/cee }\end{array}$ \\
\hline
\end{tabular}

Duarte-Gamas et al.: RDW as a Long-Term Prognostic Marker after Carotid Endarterectomy

Table 2. Survival analysis of prognostic factors

\begin{tabular}{|c|c|c|c|}
\hline & Patients, $n$ & MST, months $(95 \% \mathrm{CI})$ & $p$ value \\
\hline Age & & & 0.270 \\
\hline$<75$ years & 116 & $50(47.3-52.7)$ & \\
\hline$>75$ years & 64 & $33(11.1-54.9)$ & \\
\hline Sex & & & 0.512 \\
\hline Female & 36 & $52(19.2-84.8)$ & \\
\hline Male & 144 & $49(41.9-56.1)$ & \\
\hline Hypertension & & & 0.145 \\
\hline No & 23 & $54(18.9-89.2)$ & \\
\hline Yes & 157 & $50(43.7-56.3)$ & \\
\hline Smoking history & & & 0.306 \\
\hline No & 89 & $51(40.9-61.1)$ & \\
\hline Yes & 91 & $47(38.6-55.4)$ & \\
\hline Diabetes mellitus & & & 0.642 \\
\hline No & 102 & $50(38.8-61.3)$ & \\
\hline Yes & 78 & $50(40.5-59.5)$ & \\
\hline Dyslipidemia & & & 0.058 \\
\hline No & 25 & $57(51.8-62.2)$ & \\
\hline Yes & 155 & $49(41.1-56.9)$ & \\
\hline CKD & & & 0.001 \\
\hline No & 155 & $49(41.7-56.4)$ & \\
\hline Yes & 25 & $50(19.4-80.6)$ & \\
\hline BMI $>30$ & & & 0.447 \\
\hline No & 148 & $50(42.4-57.6)$ & \\
\hline Yes & 32 & $47(21.4-72.6)$ & \\
\hline PAD & & & 0.004 \\
\hline No & 138 & $49(40.3-57.7)$ & \\
\hline Yes & 42 & $57(34.5-79.5)$ & \\
\hline CAD & & & 0.549 \\
\hline No & 119 & $44(33.3-54.7)$ & \\
\hline Yes & 61 & $52(42.8-61.2)$ & \\
\hline COPD & & & 0.877 \\
\hline No & 157 & $51(48.2-53.8)$ & \\
\hline Yes & 23 & $28(16.6-39.4)$ & \\
\hline $\mathrm{CHF}$ & & & 0.051 \\
\hline No & 158 & 47 (38.7-55.4) & \\
\hline Yes & 22 & $80(47.0-113)$ & \\
\hline Atrial fibrillation & & & 0.101 \\
\hline No & 168 & $50(42.9-57.1)$ & \\
\hline Yes & 12 & $59(16.6-101)$ & \\
\hline Asymptomatic CS & 100 & $49(39.8-58.2)$ & 0.347 \\
\hline Symptomatic CS & 80 & $50(32.7-67.3)$ & \\
\hline \multirow[t]{2}{*}{ BB } & 117 & $50(41.6-58.4)$ & 0.984 \\
\hline & 56 & $52(45.7-58.3)$ & \\
\hline \multirow[t]{2}{*}{ ACEI } & 49 & $53(43.6-62.4)$ & 0.054 \\
\hline & 124 & $50(42.1-57.9)$ & \\
\hline \multirow[t]{2}{*}{ ССВ } & 107 & $51(47.5-54.5)$ & 0.592 \\
\hline & 66 & $42(24.159 .9$ & \\
\hline \multirow[t]{2}{*}{ Thiazide diuretics } & 96 & $51(47.3-54.7)$ & 0.926 \\
\hline & 77 & $42(26.1-57.9)$ & \\
\hline Hemoglobin & & & 0.135 \\
\hline$>13.115 \mathrm{~g} / \mathrm{dL}$ & 87 & $53(48.01-58.00)$ & \\
\hline$<13.115 \mathrm{~g} / \mathrm{dL}$ & 93 & $41(26.37-55.63)$ & \\
\hline
\end{tabular}


Table 2 (continued)

\begin{tabular}{lclc}
\hline & Patients, $n$ & MST, months $(95 \% \mathrm{CI})$ & $p$ value \\
\hline $\begin{array}{l}\text { NLR } \\
\quad 2.52\end{array}$ & & & 0.074 \\
$\quad<2.52$ & 77 & $49(39.16-58.84)$ & \\
PLR & & $50(40.37-59.63)$ & 0.609 \\
$\quad \geq 124.88$ & 95 & $51(47.08-54.92)$ & \\
$\quad<124.88$ & 85 & $44(29.3-58.70)$ & 0.097 \\
HPR & & $51(47.08-54.917)$ & \\
$\quad \geq 0.0651$ & 95 & $44(29.301-58.70)$ & \\
$<0.0651$ & 85 &
\end{tabular}

Statistically significant results are highlighted in bold. ASA, American Society of Anesthesiologists Physical Status Classification System; CAD, coronary artery disease; CS, carotid stenosis; CCB calcium channel blockers; $\mathrm{CHF}$, congestive heart failure; $\mathrm{CKD}$, chronic kidney disease (creatinine $=1.5 \mathrm{mg} / \mathrm{dL}$ ); COPD, chronic obstructive pulmonary disease; MST, median survival time; PAD, peripheral artery disease; BMI, body mass index (obesity >30).

Table 3. Multivariable analyses of prognostic factors for MACE and all-cause mortality

\begin{tabular}{llll}
\hline & aHR & $95 \%$ CI & $p$ value \\
\hline MACE & & & \\
Dyslipidemia & 0.678 & $0.355-1.296$ & 0.240 \\
CKD & 2.056 & $1.117-3.786$ & $\mathbf{0 . 0 2 1}$ \\
PAD & 1.545 & $0.869-2.745$ & 0.138 \\
CHF & 1.659 & $0.888-3.099$ & 0.112 \\
RDW-CV 13.95 & 2.047 & $1.202-3.487$ & $\mathbf{0 . 0 0 8}$ \\
Hemoglobin & 0.934 & $0.764-1.140$ & 0.501 \\
NLR & 1.057 & $0.875-1.277$ & 0.566 \\
HPR & $8.0 \mathrm{e}-6$ & $6.8953 \mathrm{e}-11$ to 1.023 & $\mathbf{0 . 0 5 0}$ \\
All-cause mortality & & & 0.466 \\
Dyslipidemia & 0.746 & $0.338-1.643$ & $\mathbf{0 . 0 0 4}$ \\
CKD & 2.904 & $1.410-5.982$ & $\mathbf{0 . 0 3 2}$ \\
PAD & 2.138 & $1.066-4.292$ & 0.121 \\
CHF & 1.810 & $0.855-3.834$ & $\mathbf{0 . 0 1 1}$ \\
RDW-CV 13.95 & 2.455 & $1.231-4.894$ & 0.896 \\
Hemoglobin (g/dL) & 0.986 & $0.795-1.223$ & 0.381 \\
NLR & 1.091 & $0.898-1.327$ & $\mathbf{0 . 0 1 9}$ \\
HPR & $2.6501 \mathrm{e}-8$ & $9.0495 \mathrm{e}-15$ to 0.078 & \\
\hline
\end{tabular}

Statistically significant results are highlighted in bold. MACE, major adverse cardiovascular events; aHR, adjusted hazard ratio; CAD, coronary artery disease; CHF, congestive heart failure; CKD, chronic kidney disease (creatinine $=1.5 \mathrm{mg} / \mathrm{dL}$ ); HPR, hemoglobin to platelet ratio; NLR, neutrophil to lymphocyte ratio; PAD, peripheral artery disease; BMI, body mass index (obesity $>30$ ); RDW-CV, red blood cell distribution width-coefficient of variation.

pendent predictor of carotid plaque development and plaque progression [20]. The present prospective cohort study has further demonstrated that RDW-CV is associated with longterm adverse outcomes after CEA, namely MI and all-cause mortality.

As expected, lower preoperative hemoglobin values were associated with higher RDW-CV. Preoperative anemia has been associated with increased postoperative mortality in patients 


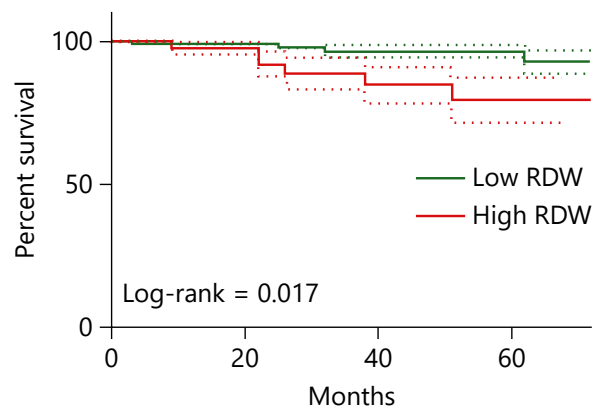

\begin{tabular}{|c|c|c|c|c|c|c|}
\hline & $30 \mathrm{~d}$ & $12 \mathrm{~m}$ & $24 \mathrm{~m}$ & $36 \mathrm{~m}$ & $48 \mathrm{~m}$ & $60 \mathrm{~m}$ \\
\hline RDW-CV & $100 \%$ & $99.1 \%$ & $99.1 \%$ & $96.4 \%$ & $96.4 \%$ & $96.4 \%$ \\
$<13.95$ & $\mathrm{SE}: 0$ & $\mathrm{SE}: 0.9 \%$ & $\mathrm{SE}: 0.9 \%$ & $\mathrm{SE}: 2.1 \%$ & $\mathrm{SE}: 2.1 \%$ & $\mathrm{SE}: 2.1 \%$ \\
& 0 & 1 & 1 & 3 & 3 & 3 \\
& 126 & 101 & 80 & 61 & 54 & 28 \\
\hline RDW-CV & $100 \%$ & $97.8 \%$ & $91.9 \%$ & $88.8 \%$ & $84.8 \%$ & $84.8 \%$ \\
$\geq 13.95$ & $\mathrm{SE}: 0$ & $\mathrm{SE}: 2.2 \%$ & $\mathrm{SE}: 4.6 \%$ & $\mathrm{SE}: 5.3 \%$ & $\mathrm{SE}: 6.4 \%$ & $\mathrm{SE}: 6.4 \%$ \\
& 0 & 1 & 3 & 4 & 5 & 4 \\
& 49 & 42 & 31 & 22 & 17 & 8 \\
\hline
\end{tabular}

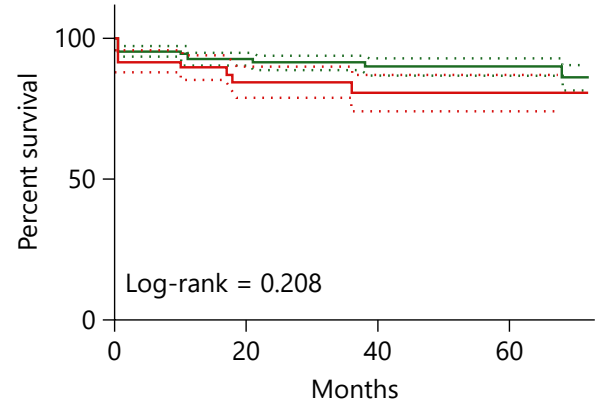

\begin{tabular}{|c|c|c|c|c|c|c|}
\hline & $30 \mathrm{~d}$ & $12 \mathrm{~m}$ & $24 \mathrm{~m}$ & $36 \mathrm{~m}$ & $48 \mathrm{~m}$ & $60 \mathrm{~m}$ \\
\hline RDW-CV & $95.4 \%$ & $92.5 \%$ & $91.3 \%$ & $91.3 \%$ & $89.8 \%$ & $89.8 \%$ \\
$<13.95$ & SE:1.8\% & SE:2.4\% & SE:2.7\% & SE:2.7\% & SE:3\% & SE:3\% \\
& 6 & 9 & 10 & 10 & 11 & 11 \\
& 120 & 93 & 75 & 61 & 53 & 28 \\
\hline RDW-CV & $91.8 \%$ & $89.7 \%$ & $84.6 \%$ & $80.8 \%$ & $80.8 \%$ & $80.8 \%$ \\
$\geq 13.95$ & SE:3.9\% & SE:4.4\% & $5.4 \%$ & SE:6.4\% & SE: $6.4 \%$ & SE: $6.4 \%$ \\
& 4 & 5 & 7 & 8 & 8 & 8 \\
& 45 & 41 & 30 & 21 & 17 & 8 \\
\hline
\end{tabular}

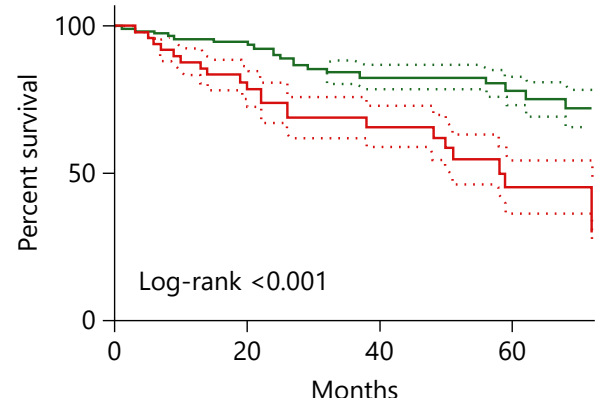

\begin{tabular}{|c|c|c|c|c|c|c|}
\hline & $30 \mathrm{~d}$ & $12 \mathrm{~m}$ & $24 \mathrm{~m}$ & $36 \mathrm{~m}$ & $48 \mathrm{~m}$ & $60 \mathrm{~m}$ \\
\hline RDW-CV & $99.2 \%$ & $94.9 \%$ & $89.6 \%$ & $83.6 \%$ & $82.2 \%$ & $77.4 \%$ \\
$<13.95$ & SE:0.8\% & SE:2\% & SE:3\% & SE:3.8\% & SE:4\% & SE:5\% \\
& 1 & 6 & 11 & 16 & 17 & 19 \\
& 126 & 101 & 79 & 61 & 54 & 28 \\
\hline RDW-CV & $100 \%$ & $87.8 \%$ & $74.0 \%$ & $69.1 \%$ & $62.3 \%$ & $45.3 \%$ \\
$\geq 13.95$ & SE:0 & SE:4.7\% & SE:6.5\% & SE:6.9\% & SE:7.8\% & SE:9.3\% \\
& 0 & 6 & 12 & 14 & 16 & 20 \\
& 49 & 42 & 31 & 22 & 17 & 8 \\
\hline
\end{tabular}

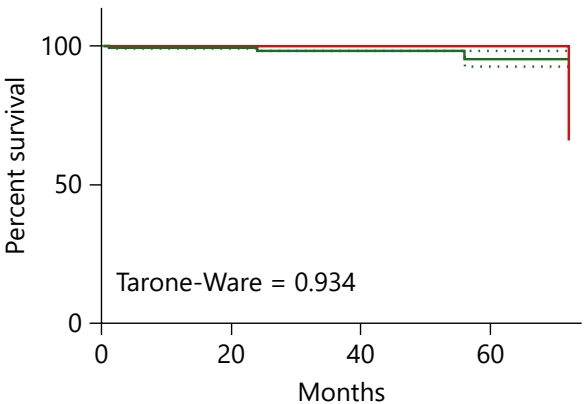

\begin{tabular}{|c|c|c|c|c|c|c|}
\hline & $30 \mathrm{~d}$ & $12 \mathrm{~m}$ & $24 \mathrm{~m}$ & $36 \mathrm{~m}$ & $48 \mathrm{~m}$ & $60 \mathrm{~m}$ \\
\hline RDW-CV & $100 \%$ & $99.2 \%$ & $98 \%$ & $98 \%$ & $98 \%$ & $95.6 \%$ \\
$<13.95$ & $\mathrm{SE}: 0$ & $\mathrm{SE}: 0.8 \%$ & $\mathrm{SE}: 1.4 \%$ & $\mathrm{SE}: 1.4 \%$ & $\mathrm{SE}: 1.4 \%$ & $\mathrm{SE}: 2.8 \%$ \\
& 0 & 1 & 2 & 2 & 2 & 3 \\
& 126 & 102 & 80 & 64 & 57 & 30 \\
\hline RDW-CV & $100 \%$ & $100 \%$ & $100 \%$ & $100 \%$ & $100 \%$ & $100 \%$ \\
$\geq 13.95$ & $\mathrm{SE}: 0$ & $\mathrm{SE}: 0$ & $\mathrm{SE}: 0$ & $\mathrm{SE}: 0$ & $\mathrm{SE}: 0$ & $\mathrm{SE}: 0$ \\
& 0 & 0 & 0 & 0 & 0 & 0 \\
& 49 & 43 & 33 & 24 & 19 & 8 \\
\hline
\end{tabular}

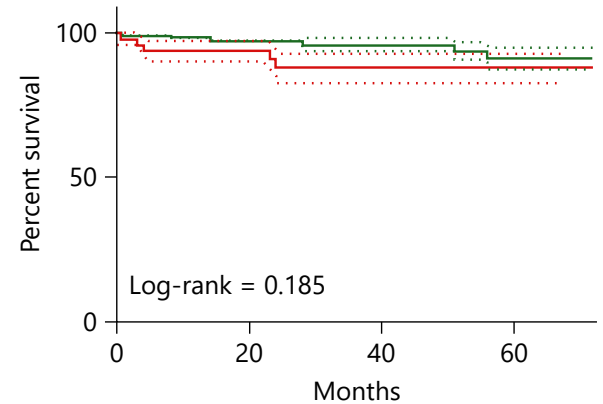

\begin{tabular}{|c|c|c|c|c|c|c|}
\hline & $30 \mathrm{~d}$ & $12 \mathrm{~m}$ & $24 \mathrm{~m}$ & $36 \mathrm{~m}$ & $48 \mathrm{~m}$ & $60 \mathrm{~m}$ \\
\hline RDW-CV & $99.2 \%$ & $98.3 \%$ & $97.3 \%$ & $96 \%$ & $96 \%$ & $91.3 \%$ \\
$<13.95$ & $\mathrm{SE}: 0.8 \%$ & $\mathrm{SE}: 1.2 \%$ & $\mathrm{SE}: 1.5 \%$ & $\mathrm{SE}: 2 \%$ & $\mathrm{SE}: 2 \%$ & $\mathrm{SE}: 3.7 \%$ \\
& 1 & 2 & 3 & 4 & 4 & 6 \\
& 125 & 100 & 80 & 62 & 55 & 28 \\
\hline RDW-CV & $98 \%$ & $93.9 \%$ & $88.0 \%$ & $88.0 \%$ & $88.0 \%$ & $88.0 \%$ \\
$\geq 13.95$ & $\mathrm{SE}: 2 \%$ & $\mathrm{SE}: 3.4 \%$ & $\mathrm{SE}: 5.1 \%$ & $\mathrm{SE}: 5.1 \%$ & $\mathrm{SE}: 5.1 \%$ & $\mathrm{SE}: 5.1 \%$ \\
& 1 & 3 & 5 & 5 & 5 & 5 \\
& 48 & 41 & 29 & 21 & 17 & 8 \\
\hline
\end{tabular}

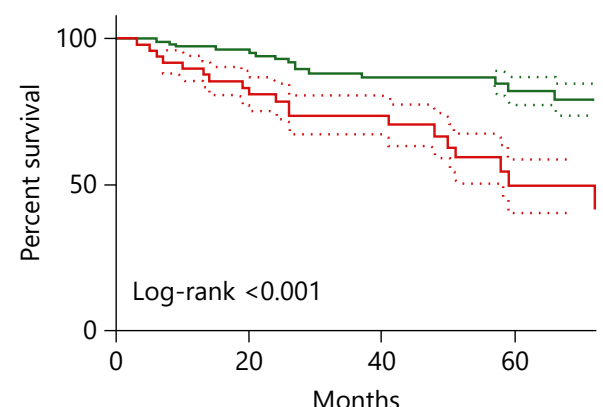

\begin{tabular}{|c|c|c|c|c|c|c|}
\hline & $30 \mathrm{~d}$ & $12 \mathrm{~m}$ & $24 \mathrm{~m}$ & $36 \mathrm{~m}$ & $48 \mathrm{~m}$ & $60 \mathrm{~m}$ \\
\hline RDW-CV & $99.2 \%$ & $96.5 \%$ & $92.3 \%$ & $87.6 \%$ & $86.2 \%$ & $81.5 \%$ \\
$<13.95$ & $\mathrm{SE}: 0.8 \%$ & $\mathrm{SE}: 1.7 \%$ & $\mathrm{SE}: 2.6 \%$ & $\mathrm{SE}: 3.4 \%$ & $\mathrm{SE}: 3.6 \%$ & $\mathrm{SE}: 4.7 \%$ \\
& 1 & 4 & 8 & 12 & 13 & 15 \\
& 126 & 102 & 81 & 64 & 57 & 30 \\
\hline RDW-CV & $100 \%$ & $87.7 \%$ & $78.5 \%$ & $73.6 \%$ & $66.5 \%$ & $49.6 \%$ \\
$\geq 13.95$ & $\mathrm{SE}: 0$ & $\mathrm{SE}: 4.7 \%$ & $\mathrm{SE}: 6.1 \%$ & $\mathrm{SE}: 6.6 \%$ & $\mathrm{SE}: 7.6 \%$ & $\mathrm{SE}: 9.4 \%$ \\
& 0 & 5 & 10 & 12 & 4 & 18 \\
& 49 & 43 & 33 & 24 & 18 & 8 \\
\hline
\end{tabular}


Duarte-Gamas et al.: RDW as a Long-Term Prognostic Marker after Carotid Endarterectomy

submitted to CEA [21]. It could be argued that hemoglobin is a confounding factor in the association between increased RDW-CV and increased mortality, since it is an early indicator of impaired erythrocyte production, particularly in iron deficiency, the most common cause of anemia [22]. However, the association between RDW-CV and long-term mortality and MACE rate remained significant, even after adjustment for hemoglobin levels, suggesting the interaction of independent pathophysiological pathways.

The pathophysiological mechanisms that link RDW-CV to atherosclerosis and cardiovascular events are not entirely elucidated. Nevertheless, some hypotheses have been proposed. Reduced iron reserves, independently of hemoglobin levels, are associated with increased RDW-CV and could play a role in atherogenesis [23]. Inflammation mechanisms could explain both increased RDW-CV and cardiovascular events. Higher RDW-CV values are independently associated with raised inflammatory parameters [24] and it is also well established that inflammation is a critical factor in plaque progression and vulnerability. As such, some authors consider RDW a marker of inflammation [23].

In the present study, atrial fibrillation was significantly more prevalent in patients with increased RDW-CV values. This association has been demonstrated previously [25]. Chronic inflammation may be the link between increased RDW-CV and the onset of atrial fibrillation. Other contributing factors may include: oxidative stress, which is associated with myofibrillar protein and cardiomyocyte membrane lipids oxidation; reduced deformability of anisocytic erythrocytes and their relationship with atrial fibrosis and arrhythmia onset, and increased cholesterol content in highly variable erythrocytes, thus contributing to atherosclerosis, a known risk factor for atrial fibrillation [26].

An interesting, relevant finding of this study was the independent association between the HPR and long-term all-cause mortality. This parameter has been assessed in some studies, mainly concerning urological neoplasms and colon and rectal cancer [27, 28]. In a study enrolling 6,046 patients submitted to percutaneous coronary intervention, HPR was independently associated with long-term MACE and cardiac and all-cause mortality [29]. Combining both hematological parameters with known biological plausibility could explain the clinical utility observed in the multivariate Cox regression model. A high count of reticulated platelets, indicative of higher platelet turnover and production, was independently associated with post-CEA myocardial injury and postoperative mortality [30]. An increased platelet count has been shown to be independently associated with mortality in both ischemic heart disease patients and population-based cohorts [31].

Moreover, CKD was associated with the long-term occurrence of MACE and mortality in this study. These results are in agreement with previous reports, which demonstrates that at 5 years CKD patients submitted to CEA have a higher incidence of coronary events and higher mortality rates [32]. Since patients with renal impairment have been associated with increased RDW-CV [33], one could argue that RDW-CV is merely a correlative factor in the relationship between CKD and long-term MACE and mortality. Yet, after multivariate analysis, the association between RDW-CV and the outcomes of interest remained significant, which suggests that RDW-CV is associated with mortality and cardiovascular events through a different pathway. Notwithstanding, an interaction and a common pathway between these 2 factors

Fig. 1. Survival plots. Sixty-month follow-up Kaplan-Meyer curves for different outcomes following CEA for groups with or without increased RDW-CV. Freedom from AMI (a), AHF (b), stroke (c), MALE (d), MACE (e), and all-cause mortality (f) after CEA according to RDW-CV. Survival tables display the percent freedom from an event (1st row), standard error (SE; 2nd row), number of events (3rd row), and number of subjects free from an event (4th row). RDW-CV, red cell distribution width-coefficient of variation; MACE, major adverse cardiovascular event; MALE, major adverse limb events; AMI, acute myocardial infarction; AHF, acute heart failure; d, days; m, months. 
are not to be excluded. Lower circulating levels of erythropoietin and chronic inflammation are both characteristics of CKD. Since both are also associated with increased RDW-CV, they may be the link between RDW, CKD, and the adverse outcomes after CEA [24].

Thefindings of this study could provide newinsights for preoperative patientmanagement. An increased RDW-CV, even in the presence of average hemoglobin values, could prompt an assessment of iron reserves, folic acid, or vitamin B12 and their timely correction. However, we found no evidence on these interventions in the literature. On the other hand, this parameter could be included in a prognostic scoring system with other independent predictors of poor survival after CEA, such as CKD [34], pre-existing CAD, and older age [35].

The main strength of the present study is the extended follow-up of the patients. Despite its prospective design, it has some limitations since it is a post hoc analysis. Patients were treated and followed in a single tertiary care center, which may not be representative of other healthcare centers. Our sample also includes both symptomatic and asymptomatic patients submitted to CEA. Although the results may be generalized to both symptomatic and asymptomatic patients, the same may not be applicable to one or another subset in particular. Further studies are needed in order to validate further the role of preoperative RDW in the management of patients with asymptomatic CS.

This study was performed in a large academic teaching institution, which might affect the external validity of the results for community hospitals that perform a large number of CEAs. The proposal of patients for surgery could represent a selection bias since subjective frail patients are less likely to be selected for invasive surgical procedures, thus inducing bias in the selected sample.

Concerning RDW, the quantification is rapid, easy, inexpensive, and it does not require specific skills or instrumentation. However, different approaches are used for measuring erythrocyte size (i.e., impedance or optical techniques), and for RDW calculation [36]. Therefore, reference values are instrument dependent and may be population dependent. There is still uncertainty concerning the real added value of RDW as a prognostic tool on top of other established cardiovascular risk factors. It is not clear if the same findings could be generalized to other institutions or different patient characteristics. Further studies are needed to find a role in the clinical practice of these findings.

\section{Conclusion}

RDW-CV is a widely available, easy to measure, and low-cost marker that independently predicts long-term mortality, MACE, and MI after CEA. Even though the mechanisms underlying these associations are not yet fully understood, this factor could prove useful in assessing which patients would likely benefit from CEA in the long term. Future research on this parameter should focus on a possible molecular basis, and interactions with inflammatory pathways, in order to shed light on the pathophysiologic mechanisms.

\section{Statement of Ethics}

The study protocol was approved by the Ethics Committee, Comissão de Ética para a Saúde do Centro Hospitalar São João (CESCHSJ), and respects the Declaration of Helsinki. This study is registered with the ClinicalTrial.gov public website with the identifier NCT04347785.

Written informed consent for data collection was not required by the CESCHSJ. Given the nature of the study, there were no benefits, risks, or discomfort for patients. The information collected was entered anonymously in a duly protected database, with the confidentiality and 
privacy of the data guaranteed. Since this study was an observational, prospective, cohort study, with the aim of evaluating the long-term and short-term prognosis of patients, with no intervention being carried out, the CESCHSJ accepted the registration of the cohort with informed consent. Most patients are still under follow-up and are completely aware of their rights regarding personal data and the registry, including the right to have their data removed from the registry. If deemed necessary, the authors are able to provide the ethics protocol.

\section{Conflict of Interest Statement}

The authors have no conflicts of interest to disclosure.

\section{Funding Sources}

This study received no external funding.

\section{Author Contributions}

Dr. Luís Duarte-Gamas contributed with the writing of the manuscript and scientific input. Dr. António Pereira-Neves contributed with data collection and scientific input. Dr. Filipa Jácome and Dr. Ricardo P. Vaz contributed with manuscript revision and scientific input. Dr. Mariana Fragão-Marques contributed with statistical analysis, scientific input, and manuscript revision. Dr. José Paulo Andrade contributed with the final manuscript revision and important scientific input. Dr. João P. Rocha-Neves contributed with the study design, statistical analysis, important scientific input, and manuscript revision.

\section{References}

1 Ferguson GG, Eliasziw M, Barr HW, Clagett GP, Barnes RW, Wallace MC, et al. The North American Symptomatic Carotid Endarterectomy Trial: surgical results in 1415 patients. Stroke. 1999 Sep;30(9):1751-8.

2 Randomised trial of endarterectomy for recently symptomatic carotid stenosis: final results of the MRC European Carotid Surgery Trial (ECST). Lancet. 1998 May;351(9113):1379-87.

3 Naylor AR, Ricco JB, de Borst GJ, Debus S, de Haro J, Halliday A, et al.; Esvs Guidelines Committee; Esvs Guideline Reviewers. Editor's Choice - Management of Atherosclerotic Carotid and Vertebral Artery Disease: 2017 Clinical Practice Guidelines of the European Society for Vascular Surgery (ESVS). Eur J Vasc Endovasc Surg. 2018 Jan;55(1):3-81.

4 Walker MD. Endarterectomy for asymptomatic carotid artery stenosis. Executive Committee for the Asymptomatic Carotid Atherosclerosis Study. JAMA. 1995 May;273(18):1421-8.

5 Halliday A, Harrison M, Hayter E, Kong X, Mansfield A, Marro J, et al.; Asymptomatic Carotid Surgery Trial (ACST) Collaborative Group. 10-year stroke prevention after successful carotid endarterectomy for asymptomatic stenosis (ACST-1): a multicentre randomised trial. Lancet. 2010 Sep;376(9746):1074-84.

6 Grobben RB, Vrijenhoek JE, Nathoe HM, Den Ruijter HM, van Waes JA, Peelen LM, et al. Clinical Relevance of Cardiac Troponin Assessment in Patients Undergoing Carotid Endarterectomy. Eur J Vasc Endovasc Surg. 2016 Apr;51(4):473-80.

7 Vilariño-Rico J, Pita-Fernández S, Segura-Iglesias RJ. Clinical predictors of major adverse cardiovascular events during long-term follow-up after carotid endarterectomy. Ann Vasc Surg. 2015 Apr;29(3):419-25.

8 Lippi G, Plebani M. Red blood cell distribution width (RDW) and human pathology. One size fits all. Clin Chem Lab Med. 2014 Sep;52(9):1247-9.

9 Uchida T. Change in red blood cell distribution width with iron deficiency. Clin Lab Haematol. 1989;11(2): 117-21.

10 Patel HH, Patel HR, Higgins JM. Modulation of red blood cell population dynamics is a fundamental homeostatic response to disease. Am J Hematol. 2015 May;90(5):422-8. 
11 Tonelli M, Wiebe N, James MT, Naugler C, Manns BJ, Klarenbach SW, et al. Red cell distribution width associations with clinical outcomes: A population-based cohort study. PLoS One. 2019 Mar;14(3):e0212374.

12 Poludasu S, Marmur JD, Weedon J, Khan W, Cavusoglu E. Red cell distribution width (RDW) as a predictor of long-term mortality in patients undergoing percutaneous coronary intervention. Thromb Haemost. 2009 Sep; 102(3):581-7.

13 Ye WY, Li J, Li X, Yang XZ, Weng YY, Xiang WW, et al. Predicting the one-year prognosis and mortality of patients with acute ischemic stroke using red blood cell distribution width before intravenous thrombolysis. Clin Interv Aging. 2020 Feb;15:255-63.

14 Saba L, Mallarini G. A comparison between NASCET and ECST methods in the study of carotids: evaluation using Multi-Detector-Row CT angiography. Eur J Radiol. 2010 Oct;76(1):42-7.

15 Agha R, Abdall-Razak A, Crossley E, Dowlut N, Iosifidis C, Mathew G, et al.; STROCSS Group. STROCSS 2019 Guideline: strengthening the reporting of cohort studies in surgery. Int J Surg. 2019 Dec;72:156-65.

16 Conte MS, Geraghty PJ, Bradbury AW, Hevelone ND, Lipsitz SR, Moneta GL, et al. Suggested objective performance goals and clinical trial design for evaluating catheter-based treatment of critical limb ischemia. J Vasc Surg. 2009 Dec;50(6):1462-73.e1-3.

17 Chen CM, Lee M, Yang YH, Huang SS, Lin CH. Association between clinical and laboratory markers and 5-year mortality among patients with stroke. Sci Rep. 2019 Aug; 9(1):11521.

18 Ani C, Ovbiagele B. Elevated red blood cell distribution width predicts mortality in persons with known stroke. J Neurol Sci. 2009 Feb;277(1-2):103-8.

19 Ren D, Wang J, Li H, Li Y, Li Z. Red blood cell distribution width and carotid intima-media thickness in patients with metabolic syndrome. BMC Cardiovasc Disord. 2017 Jan;17(1):44.

20 Lappegård J, Ellingsen TS, Vik A, Skjelbakken T, Brox J, Mathiesen EB, et al. Red cell distribution width and carotid atherosclerosis progression. The Tromsø Study. Thromb Haemost. 2015 Mar;113(3):649-54.

21 Pothof AB, Bodewes TC, O’Donnell TF, Deery SE, Shean K, Soden PA, et al. Preoperative anemia is associated with mortality after carotid endarterectomy in symptomatic patients. J Vasc Surg. 2018 Jan;67(1):183-190. e1.

22 Salvagno GL, Sanchis-Gomar F, Picanza A, Lippi G. Red blood cell distribution width: A simple parameter with multiple clinical applications. Crit Rev Clin Lab Sci. 2015;52(2):86-105.

23 Bujak K, Wasilewski J, Osadnik T, Jonczyk S, Kołodziejska A, Gierlotka M, et al. The prognostic role of red blood cell distribution width in coronary artery disease: a review of the pathophysiology. Dis Markers. 2015;2015: 824624.

24 Lippi G, Targher G, Montagnana M, Salvagno GL, Zoppini G, Guidi GC. Relation between red blood cell distribution width and inflammatory biomarkers in a large cohort of unselected outpatients. Arch Pathol Lab Med. 2009 Apr;133(4):628-32.

25 Güngör B, Özcan KS, Erdinler İ, Ekmekçi A, Alper AT, Osmonov D, et al. Elevated levels of RDW is associated with non-valvular atrial fibrillation. J Thromb Thrombolysis. 2014 May;37(4):404-10.

26 Lippi G, Cervellin G, Sanchis-Gomar F. Red blood cell distribution width: A marker of anisocytosis potentially associated with atrial fibrillation. World J Cardiol. 2019 Dec;11(12):292-304.

27 Mo CJ, Hu ZJ, Qin SZ, Chen HP, Huang L, Li S, et al. Diagnostic value of platelet-lymphocyte ratio and hemoglobin-platelet ratio in patients with rectal cancer. J Clin Lab Anal. 2020 Apr;34(4):e23153.

28 Tang G, Zhen Y, Xie W, Wang Y, Chen F, Qin C, et al. Preoperative hemoglobin-platelet ratio can significantly predict progression and mortality outcomes in patients with T1G3 bladder cancer undergoing transurethral resection of bladder tumor. Oncotarget. 2018 Jan;9(26):18627-36.

29 Zheng YY, Wu TT, Chen Y, Hou XG, Yang Y, Zhang JY, et al. Platelet-to-hemoglobin ratio as a novel predictor of long-term adverse outcomes in patients after percutaneous coronary intervention: A retrospective cohort study. Eur J Prev Cardiol. 2019 Oct;2047487319870346.

30 Meershoek AJ, Leunissen TC, van Waes JA, Klei WA, Huisman A, de Groot MC, et al. Reticulated Platelets as Predictor of Myocardial Injury and 30 Day Mortality After Non-cardiac Surgery. Eur J Vasc Endovasc Surg. 2020 Feb;59(2):309-18.

31 Goliasch G, Forster S, El-Hamid F, Sulzgruber P, Meyer N, Siostrzonek P, et al. Platelet count predicts cardiovascular mortality in very elderly patients with myocardial infarction. Eur J Clin Invest. 2013 Apr;43(4):33240.

32 Avgerinos ED, Go C, Ling J, Makaroun MS, Chaer RA. Survival and long-term cardiovascular outcomes after carotid endarterectomy in patients with chronic renal insufficiency. Ann Vasc Surg. 2015 Jan;29(1):15-21.

33 Lippi G, Targher G, Montagnana M, Salvagno GL, Zoppini G, Guidi GC. Relationship between red blood cell distribution width and kidney function tests in a large cohort of unselected outpatients. Scand J Clin Lab Invest. 2008;68(8):745-8.

34 Alves-Ferreira J, Rocha-Neves J, Dias-Neto M, Braga SF. Poor long-term outcomes after carotid endarterectomy: a retrospective analysis of two portuguese centers. Scand Cardiovasc J. 2019 Oct;53(5):266-73.

35 Imahori T, Hosoda K, Fujita A, Yamamoto Y, Mizowaki T, Miyake S, et al. Long-term outcomes of carotid endarterectomy and carotid artery stenting for carotid artery stenosis: real-world status in Japan. J Stroke Cerebrovasc Dis. 2016 Feb;25(2):360-7.

36 Fava C, Cattazzo F, Hu ZD, Lippi G, Montagnana M. The role of red blood cell distribution width (RDW) in cardiovascular risk assessment: useful or hype? Ann Transl Med. 2019 Oct;7(20):581. 